\title{
LE Dossier
}

Colette Le Petitcorps

\section{DISSIDENCE DANS LE SERVICE DOMESTIQUE. \\ L'ÉCONOMIE MORALE DE FEMMES, BONNES À L'îlLE MAURICE}

La main-d'œuvre féminine employée au service domestique est devenue l'un des facteurs de production du développement immobilier qui accompagne la restructuration de l'économie sucrière mauricienne, en particulier sur la côte. Cet article interroge la façon dont les transformations du travail et de l'espace sont appréhendées par les employées domestiques habitantes de la côte à partir des enjeux liés à la reproduction de leur foyer. L'analyse de leur économie morale dévoile un sujet politique original autour du service domestique qui déborde le champ de l'emploi.

En 2018, le gouvernement mauricien accorde des permis de construire à des investisseurs privés locaux et étrangers pour des projets de développement immobilier, commercial et hôtelier d'une valeur de plus de 54 milliards de roupies ${ }^{1}$. Par cet acte politique, l'État donne un coup d'accélérateur à son agenda de développement du territoire national qui consiste, depuis les années 2000, à convertir une grande partie des terres consacrées à la canne à sucre en zones résidentielles de luxe au profit de placements immobiliers étrangers 2 . Durant la période coloniale et jusque dans les années suivant l'indépendance (1968), les revenus extérieurs du pays provenaient exclusivement de la production du sucre, ce qui est caractéristique d'une économie de plantation ${ }^{3}$. L'activité de l'industrie sucrière s'est d'abord progressivement réduite à partir des années 1970, puis drastiquement avec la révocation en 2005 du Protocole

\footnotetext{
1. Publication officielle du gouvernement de la République de Maurice, GNews, 14 décembre 2018, p. 4.1 euro équivaut à 40 roupies mauriciennes.

2. Cette recherche a été conduite dans le cadre du projet ERC Advanced Grant: 2015 - 695573 «The Colour of Labour. The Racialised Lives of Migrants» dirigé par Cristiana Bastos à l'Institut des sciences sociales de Lisbonne, lequel interroge les processus de racialisation au travail. Je souhaite remercier Cristiana Bastos et l'équipe de recherche qui ont permis la réalisation de ce travail.

3. L'île Maurice a d'abord été sous domination coloniale hollandaise (1638-1710), puis française (1715-1810) et anglaise (1810-1968). L'économie de plantation agricole a véritablement commencé sous la domination coloniale anglaise, d'abord sous le régime de l'esclavage, puis sous celui de l'engagisme. Voir notamment V. Teelock, Bitter Sugar: Sugar and Slavery in 19th Century Mauritius, Moka (île Maurice), Mahatma Gandhi Institute, 1998.
} 
Politiser le travail domestique

Sucre entre les pays ACP (Afrique, Caraibes, Pacifique) et l'Union européenne, lequel protégeait le sucre mauricien de la concurrence internationale ${ }^{4}$. Pour les anciens groupes sucriers et l'État, la transformation radicale du système productif ne devait pas stopper le processus d'«optimisation des ressources en terres $^{5}$ » pour l'accumulation du capital ${ }^{6}$. C'est dans le cadre de la poursuite de cet objectif que le développement immobilier de luxe a été étendu à l'ensemble du territoire. La continuité du projet capitaliste s'opère néanmoins avec des changements profonds dans l'usage de la force de travail et de la terre.

Le développement immobilier requiert de la main-d'œuvre pour la construction des résidences mais, sur le long terme, il crée essentiellement des emplois domestiques 7 . Aussi ancien que le travail de laboureur ${ }^{8}$ (ouvrier agricole) dans les champs de canne, le service domestique est, à l'île Maurice, exercé à $87 \%$ par des femmes, selon le dernier recensement de la population de 2011. Il demeure en partie dans l'économie informelle, comme dans bien d'autres pays du monde, et s'effectue aujourd'hui le plus souvent à temps partiel: bonnes, cuisinières, nounous ou garde-malades travaillent généralement plusieurs heures par jour (ou par semaine) pour le même employeur particulier et cumulent parfois plusieurs travails auprès de différents employeurs. La mise en valeur du «service à la Mauricienne » prévu pour l'entretien des logements de luxe participe parfois aux procédés par lesquels les étrangers sont incités à investir dans l'immobilier'9. L'argument de vente du service repose sur le message implicite que les femmes mauriciennes auraient incorporé une déférence caractéristique du service colonial et hérité de l'esclavage de plantation. Les femmes employées au service domestique sont également intégrées aux procédés de constitution des profits des acheteurs immobiliers lorsque ceux-ci mettent leur bien en location. La qualité du service à la personne est en effet évaluée, notée et commercialisée sur TripAdvisor ou Airbnb. Le service domestique contribue donc à la mise en valeur du capital et devient en ce sens productif ${ }^{10}$. Il circule dans l'espace marchand

4. Le Protocole avait été signé en 1975 à la Convention de Lomé.

5. M.-L. Lam Hung, Les biens immobiliers à Maurice. Acquisition - succession - fiscalité, Maurice, T-Printers, 2010, p. 18.

6. U. Kothari et R. Wilkinson, «Global Change, Small Island State Response: Restructuring and the Perpetuation of Uncertainty in Mauritius and Seychelles ", Journal of International Development, vol. $25, \mathrm{n}^{\circ} 1,2013$, p. 92-107.

7. Le mouvement socialiste Lalit (la lutte) a renseigné ce point dans son magazine en ligne Revi Lalit. 8. Les termes en italique renvoient aux expressions vernaculaires de l'île Maurice dont une importante partie est tirée de la langue créole.

9. Cette expression est revenue plusieurs fois lors du «Forum des expatriés», organisé en juin 2019 par Le Monde et Courrier International à Paris, où plusieurs conseillers et promoteurs immobiliers présentaient les investissements possibles à l'île Maurice pour les Français.

10. K. Marx, «Plus-value absolue et plus-value relative», in K. Marx, Le Capital, livre I, Paris, Flammarion, 2014, chapitre 16. 
(transnational) de l'immobilier et du service domestique, des représentations racistes et sexuées sur les qualités incorporées des femmes mauriciennes au service domestique, comme des travaux en ont fait l'analyse à propos d'autres employées domestiques ailleurs dans le monde ${ }^{11}$. Dans le contexte mauricien cependant, rares sont les agences de recrutement et les dispositifs de formation s'assurant de l'adéquation effective de la main-d'œuvre à cette image commerciale et postcoloniale. Le marché du service domestique repose encore en grande partie sur la mise en relation informelle des bonnes et des employeurs.

Au lieu d'analyser la façon dont les femmes employées domestiques mauriciennes se conforment ou non à l'image marchande de leur aptitude au service dévoué, j'interroge le sens que celles-ci donnent aux transformations des usages de la terre et de la force de travail dans le contexte économique actuel. Ces transformations affectent non seulement leur travail de bonne, mais aussi les conditions matérielles d'existence de leur foyer. Cette question a émergé au cours de l'enquête de terrain que j'ai menée entre 2017 et 2019 sur la côte sud-ouest de l'île Maurice, entre les villages de Tamarin et Rivière Noire, où j'ai suivi une trentaine de femmes employées comme bonnes dans leurs espaces d'habitat (à leur domicile et dans les lieux de rencontre entre villageois) ${ }^{12}$. Dans le district de Rivière Noire dont fait partie cet espace, le service domestique concentre $16 \%$ de l'emploi féminin de la région, contre une moyenne nationale de $9 \% 13$. Le développement résidentiel de luxe pour Mauriciens et étrangers y est particulièrement avancé. Il a brutalement débuté entre Tamarin et Rivière Noire à la fin des années 1990 alors que la région était jusque-là rurale. Bien que cet espace côtier soit réputé pour être peuplé par des Créoles, associés dans les représentations aux descendants d'esclaves, j'ai rencontré autant de femmes créoles que de femmes hindoues parmi les employées domestiques. Ces dernières sont affiliées aux descendants d'engagés indiens, d'après la catégorisation ethnique qui est toujours en vigueur dans le sens commun et sur le plan politique ${ }^{14}$. La plupart de ces femmes travaillent actuellement comme bonnes, mais j'en ai également

\footnotetext{
11. J. Debonneville, «Les écoles du care aux Philippines. Le devenir travailleuse domestique au prisme de l'altérité», Revue Tiers Monde, n²17, 2014, p. 61-78; F. Scrinzi, Genre, migrations et emplois domestiques en France et en Italie. Construction de la non-qualification et de l'altérité ethnique, Paris, Éditions Petra, 2013.

12. L'enquête de terrain a duré dix mois et s'est étalée sur quatre périodes. Les femmes rencontrées sont nées sur la côte ou y habitent depuis au moins vingt ans.

13. D'après le recensement de la population de 2011 effectué par le Statistic Board.

14. Une ample littérature a traité de la construction des catégories ethniques de classement de la population et de leurs usages politiques à l'île Maurice. Voir, par exemple, S. Chazan-Gillig et P. Ramhota, L'hindouisme mauricien dans la mondialisation. Cultes populaires indiens et religion savante, Marseille/Paris/Moka, IRD/Karthala/MGI, 2009.
} 
Politiser le travail domestique

rencontré quelques-unes qui ont travaillé dans le service domestique du temps de l'économie sucrière précédant le développement immobilier de la zone.

En adoptant la démarche de Gérard Althabe pour la conduite de mon enquête, j'ai cherché à appréhender la manière dont les sujets femmes employées domestiques produisaient un sens à leur monde et à leurs rapports sociaux ${ }^{15}$. J'ai donc étudié leur rapport au service domestique en partant de ce qui constituait le «centre de leur narration ${ }^{16}$ » : une préoccupation constante vis-à-vis de la survie de leur foyer. Ce point de départ de l'analyse me conduit ici à procéder à l'examen de l'économie morale ${ }^{17}$ par laquelle mes interlocutrices jugent les conditions du service domestique que leurs employeurs actuels veulent leur imposer ${ }^{18}$. J'explore dans un premier temps leurs normes et leurs conceptions sociales de la justice au travail en partant de leurs mémoires des rapports de dépendance qui étaient à l'œuvre dans un passé récent où l'économie de plantation prédominait. Je rends compte dans un second temps de la perte de légitimité, à leurs yeux, de l'autorité de l'ensemble de ceux qui bénéficient actuellement de leurs services pour des usages personnels et/ou lucratifs (employeurs particuliers, propriétaires terriens, promoteurs immobiliers et État). J'étudie pour finir la façon dont cette dissidence vis-à-vis des bénéficiaires du service domestique, dans le contexte du développement immobilier, est à l'origine d'actions collectives sur leurs conditions de travail, dont l'enjeu politique diffère de celui qui vise la transformation du service domestique en travail salarié ${ }^{19}$. La démarche suivie se distingue donc des études qui appréhendent le service domestique contemporain en le situant dans la reproduction linéaire des rapports de domination, de genre et de race hérités de l'époque coloniale et esclavagiste ${ }^{20}$. En m'efforçant de «reconstruire

15. G. Althabe, «Vers une ethnologie du présent», in G. Althabe et M. Selim, Démarches ethnologiques au présent, Paris, L’Harmattan, 1998, p. 9-36.

16. Expression de Fava à propos de la démarche d'enquête d'Althabe à Madagascar. F. Fava, Qui suis-je pour mes interlocuteurs? L'anthropologue, le terrain et les liens émergents, Paris, L'Harmattan, 2014, p. 48.

17. J. C. Scott, The Moral Economy of the Peasant: Rebellion and Subsistence in South East Asia, New Haven/Londres, Yale University Press, 1976.

18. J'ai également rencontré une vingtaine d'employeurs, mauriciens et étrangers, lors de l'enquête de terrain. Leurs discours ne font toutefois pas directement l'objet de cet article.

19. Dans ce sens, j'inscris ma démarche dans la lignée de l'approche de J. Siméant, «Protester/ mobiliser/ne pas consentir. Sur quelques avatars de la sociologie des mobilisations appliquée au continent africain", Revue internationale de politique comparée, vol. 20, n², 2013, p. 138.

20. Voir par exemple E. N. Glenn, «De la servitude au travail de service: les continuités historiques de la division raciale du travail reproductif payé», in E. Dorlin (dir.), Sexe, race, classe, pour une épistémologie de la domination, Paris, PUF, 2009, p. 21-70; J. Rollins, «Entre femmes. Les domestiques et leurs patronnes", Actes de la recherche en sciences sociales, n 84, 1990, p. 63-77. 
l'univers des sujets ${ }^{21}$ » femmes employées domestiques que j'ai rencontrés, je vise au contraire à dévoiler les déplacements du sens et des enjeux du travail que celles-ci infligent au marché des emplois domestiques, ce qui contraint les acteurs impliqués dans le développement immobilier à revoir leurs stratégies d'exploitation de la force de travail.

\section{LE " TRAVAIL DANS LA COUR " OU LA MÉmOIRE DES ARRANGEMENTS SOCIAUX DE LA CÔTE}

Pauline: «À La Preneuse, y avait... 34 maisons de Blancs mauriciens, qui sont venus de loin, qui sont venus à Maurice pour développer l'île Maurice. Et beaucoup sont descendus de Curepipe, pour venir à Rivière Noire faire un petit campement pour passer le moment de l'hiver. Et c'est eux qui ont commencé à construire Rivière Noire, construire un peu le développement de la côte. Quand ils sont venus, là ils ont pris les gens d'ici, le monsieur va à la pêche, la dame pour travailler comme bonne, et ils ont fait, à l'arrière de la cour, une 'tite maisonnette qu'on appelait la dépendance. Pour que les autres personnes habitent dedans avec eux ${ }^{22}$.»

Dans son récit du peuplement des villages côtiers de La Preneuse et de Rivière Noire, Pauline livre une description subjective du rapport de dépendance qui liait autrefois tous les travailleurs, hommes et femmes, aux propriétaires des cours de la zone désignés comme «Blancs mauriciens». Née dans une dépendance dans les années 1950, elle s'appuie sur ses souvenirs de jeunesse pour préciser ce qui constituait, dans les années 1960, 1970, jusqu'aux années 1980, les conditions matérielles du travail dans la cour, selon l'expression de la langue créole: on y travaillait autant qu'on y vivait. Comme elle en témoigne, la cour, située soit sur des terrains privés, soit sur des terres louées en bail à l'État ${ }^{23}$, ne constituait pas seulement des lieux de villégiature des Blancs, mais également de petites unités de production organisées autour de la pêche. Il n'y avait pas seulement des femmes qui y travaillaient comme bonnes, mais également des hommes qui exerçaient souvent les trois fonctions réunies de pêcheur, gardien de campement et jardinier. L'unité de production de la cour incluait à la fois du travail productif (pêche et parfois vente de ses produits) et du travail reproductif (entretien du campement et de

21. P. Absi, L. Bazin et M. Selim, «Les dominations enchevêtrées. Investissements épistémologiques de l'anthropologie du travail», L'Homme et la société, n 193-194, 2014, p. 153-180.

22. Entretien avec Pauline, Rivière Noire, 20 juillet 2018.

23. Les 81 mètres du bord de mer tout autour de l'île sont la propriété de l'État depuis la loi des Pas géométriques de l'arrêté Decaen de 1807. 
Politiser le travail domestique

ses habitants) ${ }^{24}$. Le travail était à la fois rémunéré en argent et en nature, à travers l'octroi d'un logement au pêcheur, à la bonne et à leur famille, ainsi que par le partage de la nourriture issue de la pêche et de la chasse. D'après les mots de Pauline, «les gens d'ici», dont elle fait partie, n'avaient pas d'existence matérielle, ni idéelle, sur la côte sans les propriétaires des cours. Ces derniers détenaient les moyens de production (la terre et le bateau) ainsi que l'usage de la force de travail. Outre les cours, cette région côtière était occupée par les unités de production plus larges des salines, des champs de cannes et des terrains de chasse que les travailleurs appelaient tablisman (établissement), terme employé à l'origine pour caractériser la plantation sucrière ${ }^{25}$. À l'instar de la cour, le mot désignait autant la propriété des Blancs que l'espace de travail et de vie des travailleurs et de leur famille. Dans les mémoires relatées par les femmes rencontrées, le travail dépeint de l'époque n'est pas défini en premier lieu par la tâche spécifique exécutée, mais par le groupe social auquel son produit appartenait. Dans les discours, la bonne est ainsi incluse au sein de l'ensemble des travailleurs au service des propriétaires des cours et des tablisman dont l'appartenance est racisée: il s'agissait des Blancs.

En échange du fait que la cour fournissait les principales conditions matérielles d'existence du foyer de ses travailleurs, ces derniers devaient au propriétaire un travail qui n'avait pas de limites précises, que ce soit pour les tâches à effectuer ou pour le temps à y consacrer. La bonne était souvent l'épouse du pêcheur qui gardait le campement tout au long de l'année. Alors qu'elle était moins payée que son mari, son service était conçu comme devant se faire en échange du droit de vivre dans la dépendance. Quand la famille du propriétaire venait y passer ses vacances ou le week-end, elle pouvait alors être sollicitée pour des services variés : descendre les bagages, ouvrir la porte, servir un verre d'eau, laver les pieds, brosser les cheveux, découvrir le couvrelit pour le coucher, etc. «C'était une condition», comme le rappelle Marie qui travaillait dans la cour à Rivière Noire dans les années 1970:

Marie $^{26}$ : «Madame me dit, comme Mario [son mari] travaillait comme gardien et que, comment dire, on restait dans la dépendance, quand Madame avait besoin de moi je devais y aller. Parce qu'étant donné qu'ils nous donnaient la maison, j'avais besoin, comment dire, au moment où elle a besoin de moi, je dois y aller. C'était une condition, c'était comme ça.

24. Cette approche matérialiste du travail dans la cour est inspirée du concept de "plantation household» conçu par Elizabeth Fox-Genovese pour décrire les rapports de production et de reproduction de la société esclavagiste du Sud américain avant la guerre de Sécession. Voir E. Fox-Genovese, Within the Plantation Household: Black and White Women of the Old South, Chapel Hill/Londres, The University of North Carolina Press, 1988.

25. M. Claveyrolas, Quand l'hindouisme est créole. Plantation et indianité à l'île Maurice, Paris, Éditions de l'EHESS, 2017.

26. Les extraits d'entretiens à partir de celui-ci sont traduits du créole. 
Vous n'étiez pas payée?

Elle me payait mais seulement, vous savez, pas grand-chose, une petite pièce.

Vous faisiez le ménage...

Oui, je faisais servante ${ }^{27}$.»

Dans tous ses faits et gestes, la bonne ou la servante devait adopter dans la maison une attitude servile face à la Madame, au Misie (Monsieur) et à leurs enfants, comme mes interlocutrices en font l'analyse rétrospective. Le fait qu'il leur était interdit d'utiliser les toilettes du campement est, par exemple, un des souvenirs des femmes ayant servi dans les années 1960, 1970 et 1980. Celles-ci soulignent ainsi les rituels interpersonnels ${ }^{28}$ au sein de l'espace domestique qui visaient à leur faire intérioriser leur infériorité raciale, d'après l'idéologie constitutive de la hiérarchie sociale de la société coloniale et postcoloniale de cette époque.

D'après les propos des femmes de l'enquête, le service domestique sans limites en termes de tâches ou de temps de travail, ainsi que l'attitude servile exigée de la bonne étaient néanmoins subis dans l'objectif d'obtenir en échange les ressources matérielles nécessaires à la subsistance de leur foyer. Les conditions matérielles d'existence de leur foyer, confiné dans l'espace de la cour, configuraient leur dépendance au travail de service domestique et à ses formes serviles. Mais cela s'assortissait de l'élaboration d'une norme sociale de ce que devait être, selon elles, «la condition » réciproque du travail dans la cour: en échange de la déférence, elles attendaient un certain nombre de ressources matérielles pour le maintien en vie et la reproduction de leur foyer, telles que la nourriture, mais aussi par exemple une aide relative aux frais de santé. Ces ressources devenaient un droit de leur point de vue, ainsi qu'un critère de jugement des bons et des mauvais propriétaires. Les femmes employées comme bonnes participaient donc à l'institution d'une économie morale du rapport de dépendance, non pas en tant que collaboratrices de leur propre domination ${ }^{29}$, mais dans leur propre intérêt pour garantir le droit à la subsistance de leur foyer.

Les ressources pour la survie du foyer n'étaient en revanche pas uniquement obtenues directement par le travail pour les propriétaires des cours et des tablismans, d'après les récits collectés. Pour vivre selon les conditions de subsistance de l'époque, il fallait aller chercher de l'eau à la rivière, du bois pour cuire les plats sur le feu et de l'herbe pour nourrir les vaches ou les cabris élevés à proximité des maisons des travailleurs. Ces tâches exigeaient

27. Entretien avec Marie, Rivière Noire, 23 juillet 2018.

28. J. Rollins, «Entre femmes...», art. cité.

29. Selon les termes de Bourdieu pour qualifier la violence symbolique, voir P. Bourdieu, Méditations pascaliennes, Paris, Seuil, 2003. 
Politiser le travail domestique

une organisation collective entre les travailleurs où les femmes jouaient un rôle important. C'étaient elles qui allaient par petits groupes chercher ces ressources naturelles, ce qui impliquait de fait d'aller sur les territoires des Blancs qui n'étaient pas réservés aux habitations des travailleurs ${ }^{30}$. Leur pratique, sociale, était parfois tolérée par les propriétaires de la zone, et parfois interdite, comme plusieurs femmes en ont témoigné en évoquant le chassé 31 de Tamarin où elles allaient récupérer du bois. Voici ce que se rappelle Solange par exemple, à l'époque où elle avait commencé à travailler dans la cour dans les années 1970:

Solange: «Un jour, j'ai entendu un monsieur devant moi. Un gardien. Son fusil avec lui. Ben je le connaissais, je lui ai dit: "Aïo, tu ne vas pas prendre mon bois?" Il m'a dit: “Non, vous savez, vous ne devez pas revenir, parce que ce monsieur-là il est mauvais. S'il vous trouve là, il peut vous tirer une balle là." Il nous a fait peur, on a arrêté d'aller. Allé allé, j'avais besoin de passer par là-bas, j'ai cassé deux, trois bouts de bois parce qu'ils étaient trop jolis. Vous ne pouvez pas juste regarder, vous devez casser ${ }^{32}$ !»

En bravant l'interdit, les femmes habitant la côte obligeaient finalement les propriétaires des terres à s'adapter à leur organisation sociale pour la subsistance. Celle-ci se mettait en place afin de survivre aux conditions d'exploitation du travail existant à l'époque, tout en permettant de gagner une marge d'autonomie vis-à-vis du contrôle des propriétaires terriens ${ }^{33}$. Cette autonomie relative était d'autant plus possible que les Blancs qui possédaient les cours de la côte étaient la plupart du temps absents de la zone: ils vivaient principalement dans les villes de l'intérieur de l'île et ne descendaient sur le bord de mer que pour les week-ends ou les grandes vacances. Le reste du temps, les cours et les plages appartenaient aux travailleurs et à leurs enfants. Les femmes, qu'elles soient bonnes, laboureuses ou travailleuses des salines, contribuaient à l'institutionnalisation d'un droit à la subsistance inscrit dans les rapports de dépendance aux propriétaires des unités de production. Les arrangements sociaux qui liaient de manière réciproque les propriétaires terriens aux travailleurs caractérisaient à la fois un rapport de travail (de service) et un rapport négocié à l'usage des espaces côtiers. Tout ceci a disparu avec le développement immobilier de la zone.

30. Les hommes se chargeaient davantage de la pêche, de la coupe du bois et de la confection des meubles de la maison par exemple.

31. Le terrain de chasse.

32. Entretien avec Solange, Tamarin, 6 février 2019.

33. On rapproche volontiers la formation sociale née de ce contexte historique de l'économie et des institutions formées par les esclaves dans les marges de la plantation telles qu'elles sont décrites, notamment, par S. W. Mintz et R. Price, The Birth of African-American Culture: An Anthropological Perspective, Boston, Beacon Press, 1992. 


\section{TRANSFORMATIONS ÉCONOMIQUES ET SPATIALES MOTRICES DE LA DISSIDENCE DANS LE SERVICE DOMESTIQUE}

À partir des années 1990, les grands propriétaires terriens de la zone côtière entre Tamarin et Rivière Noire entament la conversion d'une partie de leurs terres agricoles et forestières en espaces constructibles pour des résidences privées. Des Mauriciens issus à la fois de l'ancienne bourgeoisie coloniale enrichie par le sucre (principalement les «Blancs» évoqués dans la partie précédente) et de la nouvelle bourgeoisie d'État née durant les années de l'indépendance (constituée autour de l'hégémonie culturelle indo-mauricienne ${ }^{34}$ ), ainsi que des expatriés viennent s'y installer. La région est aussi pionnière, au début des années 2000, en matière d'aménagement, par des propriétaires terriens locaux ou originaires d'autres régions de l'île, de résidences de luxe fermées et sécurisées destinées à des acheteurs étrangers ${ }^{35}$. La hausse de la taxe d'habitation, imposée par l'État en 2005 et en 2010, sur les Pas géométriques situés en bord de mer incite également les anciens propriétaires des cours à rentabiliser leur bail en démolissant campements et dépendances pour construire des villas modernes, souvent mises en location sur Airbnb ${ }^{36}$. Le béton et les nouveaux murs érigés ont progressivement entièrement recouvert les anciens sites productifs de la côte (champs de canne, salines, chassés, cours), ainsi que les espaces utilisés par les travailleurs pour l'organisation collective de leur subsistance.

Depuis les années 1970 et progressivement jusqu'aux années 2000, l'État a procédé à la construction de logements à bas prix en béton, regroupés en cités, sur des terres lui appartenant ou possédées par des planteurs ${ }^{37}$. Les travailleurs des cours et des tablismans sont peu à peu devenus propriétaires de petites maisons de $40 \mathrm{~m}^{2}$ qu'ils pouvaient ensuite agrandir pour leur famille grâce à des prêts accordés par l'État. Le bétonnage serré des maisons des cités a détruit la petite production de subsistance d'autrefois qui s'organisait autour de l'élevage d'animaux et de jardins. Les foyers de travailleurs se sont ainsi transformés en unités de consommation séparées des espaces de travail.

34. M. Claveyrolas, Quand l'hindouisme est créole..., op. cit.

35. Ce plan résidentiel, accessible aux étrangers qui peuvent acheter une villa d'au moins 500000 dollars, est intitulé Integrated Resort Scheme.

36. S. Chazan-Gillig et P. Ramhota, L'hindouisme mauricien dans la mondialisation..., op. cit.

37. Les premières constructions massives de logements en béton par l'État ont débuté après le passage particulièrement dévastateur du cyclone Carole en 1960. Sur la côte, l'État a aussi attribué des baux bon marché sur les Pas géométriques à des familles de pêcheurs. Voir M. Chagny, Overview of Social Housing Programs Effected in Mauritius since the 1960s by the Government, Private Sector and NGOs, UNDP Rapid Assessment Report Social Housing, 2013, <http://nh.mu/wp-content/ uploads/2016/02/Report-Overview-Social-Housing.pdf $>$, consulté le 21 novembre 2019. 
Politiser le travail domestique

Des supermarchés vendant des produits importés ont remplacé les petits commerces et accru les besoins en liquidité des familles. La réquisition de la majeure partie des terres de la zone pour le développement de résidences haut de gamme n'a eu de cesse de réduire l'extension possible des espaces d'habitat de ces foyers ayant des revenus faibles, condamnés à vivre dans des voies sans issue exiguës.

La contribution des femmes aux revenus de leur foyer a aussi beaucoup évolué. Le développement de l'industrie textile dans la Zone franche dans les années 1970 s'est fait grâce à une main-d'œuvre principalement féminine, ce qui a contribué à généraliser l'emploi féminin ${ }^{38}$. De nombreux hommes ont perdu leur emploi au cours du processus de restructuration de l'économie sucrière ${ }^{39}$, ce qui a contribué à faire se multiplier les foyers où des femmes sont les principales pourvoyeuses de ressources financières stables. Avec le ralentissement de l'industrie textile à partir des années 1990 et le développement immobilier engagé sur la côte sud-ouest, les emplois domestiques sont devenus les emplois les plus faciles d'accès pour les femmes peu scolarisées habitant la région. À la relation organique de la bonne à l'unité de production de la cour, s'est substitué le travail rémunéré à l'heure pour un ou plusieurs employeurs de service domestique (pour du ménage, de la cuisine, du repassage, de la garde d'enfants et/ou de l'aide à des personnes malades). Les bonnes, toujours plus ou moins désignées comme telles, travaillent pour l'usage personnel de leurs employeurs et parfois aussi pour les clients de leurs employeurs qui louent une de leurs propriétés immobilières par le biais d'Airbnb. Si elles sont parfois déclarées aux services de l'État par leurs employeurs, elles ne le sont quasiment jamais lorsqu'elles font le ménage ou la cuisine pour les touristes locataires en Airbnb.

Dans les nouvelles formes du travail de ce qui constitue désormais un marché des emplois domestiques, les employeurs attendent de leurs bonnes qu'elles soient efficaces, accueillantes pour les touristes et qu'elles soient à l'heure pour faire leur travail. Des habitudes du service domestique d'autrefois sont aussi conservées, telles que la fidélité de la bonne sur la durée à ses employeurs et l'exigence d'un service très extensif impliquant de faire la cuisine, le lit, la cuisine pour le chien, le rangement des placards, etc. Ces attentes ne sont pas l'apanage des employeurs mauriciens: elles s'étendent aux propriétaires de villas étrangers qui consomment la marque de fabrique

38. C. Hein, Multinational Enterprises and Employment in the Mauritian Export Processing Zone, Multinational Enterprises Programme Working Paper No. 52, Genève, ILO, 1988; N. Burn, «Mauritius», in U. Kothari et V. Nababsing (dir.), Gender and Industrialisation: Mauritius, Bangladesh, Sri Lanka, Rose Hill, Éditions de l'Océan indien, 1996, p. 33-79.

39. U. Kothari et R. Wilkinson, "Global Change, Small Island... », art. cité. 
du paradis mauricien qui leur a été vendu. L'effectivité du «service à la Mauricienne » attendu est néanmoins soumise au sens que les femmes de la région qui exercent comme bonnes attribuent à leur travail, à leurs conditions et à leurs relations de travail.

Les femmes habitant la côte qui travaillent comme employées domestiques aujourd'hui cherchent avant tout à obtenir la liquidité nécessaire à la couverture d'un ensemble de dépenses essentielles à la subsistance de leur foyer. Le premier sujet que mes interlocutrices évoquent en entretien est d'ailleurs leur paye et la façon dont elles organisent le budget de leur foyer. Voyons par exemple le discours de Shakti, 46 ans, qui habite avec ses deux enfants scolarisés (de 9 et 11 ans) et son mari aux abords d'une cité de Tamarin:

Shakti: «Avec cette Mauricienne-là, elle m’a demandé combien je voulais. Je fais quatre heures du lundi au samedi avec elle. Ben, j'ai demandé 8000 roupies, parce qu'il y a des Sud-Africains qui payent ça. Ben, elle m'a dit oui parce que je lui dis que je joue le rôle de mari et femme chez moi. Alors je demande 80 roupies de l'heure parce que je demande ce dont j'ai besoin. Moi, je sais que si je travaille quatre heures par jour, j'ai besoin de tant. Après si je trouve un autre travail, je peux alors arriver à 15000 roupies par mois. Parce que 8000 roupies Madame, ça suffit pas! Vous savez, avec 8000 roupies, si je fais ma ration tous les mois, ben tous les mois, la ration c'est 6000 ou 7000 roupies, c'est ma paye ça! Plus mes enfants vont à l'école, plus je dois leur donner leur nécessaire, leur nécessaire pour leurs leçons, c'est fini, c'est fini la paye là, vous comprenez ${ }^{40}$ ? »

Shakti évalue la rémunération qui doit correspondre à son travail en fonction des dépenses du foyer qu'elle prend en charge, ce qui dépend à la fois de sa situation familiale et de normes sociales. Son mari était soudeur, mais il ne travaille plus depuis six ans. Le foyer ne dispose donc que de son revenu de bonne. Dans les foyers de travailleurs, les femmes doivent souvent prendre en charge la ration (la nourriture achetée pour un mois), ainsi que les dépenses liées aux enfants, quelle que soit la situation du mari. Aux besoins de subsistance s'ajoutent aujourd'hui les frais de scolarité des enfants sur laquelle les femmes fondent tous leurs espoirs pour la survie du foyer ${ }^{41}$. À cela s'additionnent aussi les dépenses relatives au logement telles que l'eau, l'électricité, le loyer le cas échéant, lorsque les femmes vivent seules avec leurs enfants (ce qui représente un tiers des cas rencontrés) ou lorsque le mari est sans emploi (l'effectif des femmes comptant sur leur seul revenu correspondant

40. Entretien avec Shakti, Tamarin, 2 février 2019.

41. C'est quasiment une obligation de payer des cours particuliers pour que les élèves réussissent aux examens. Voir S. Bunwaree, "The Marginal in the Miracle: Human Capital in Mauritius", International Journal of Educational Development, vol. 21, n 3, 2001, p. 257-271. 
Politiser le travail domestique

alors à la moitié des enquêtées). L'épargne pour l'achat d'un logement n'entre même plus dans le calcul, tant l'écart entre les revenus du foyer et le coût d'achat d'un logement semble incommensurable.

La paye de bonne est donc souvent considérée comme insuffisante par rapport aux dépenses qu'elle doit couvrir, dans les conditions matérielles d'existence actuelles des foyers de travailleurs. Elle est même perçue comme injuste, si l'on écoute les propos de Shakti exprimés avec colère:

Shakti: «Je travaille six jours, pour 4000 roupies par mois. Un Mauricien, ça. Ben, il peut pas vous payer plus que ça. Ils vous disent qu'ils vous payent d'après la paye $d u$ gouvernement. Mais la paye du gouvernement, vous avez les cours particuliers des enfants à payer, on n'a pas le droit de donner la paye du gouvernement, non? Le temps que je calcule, 4000 roupies six jours par semaine pour travailler, je compte ça fait 25 roupies de l'heure. "Ah ben dites-moi Monsieur", j'ai dit, "dans 25 roupies, qu'est-ce que je gagne?" [Elle me regarde avec colère comme si j'étais le monsieur en question]. Toutes les semaines vous faites vos courses, ben votre employée elle n'a pas le droit de faire ses courses ${ }^{42}$ ?»

Par la paye du gouvernement, Shakti désigne le salaire horaire minimum fixé par la loi du travail qui régule les conditions de travail des employées domestiques depuis 1983. Un syndicat propre à ces travailleuses, le Domestic Employees Union, avait même contribué à la mise en place de cette Domestic Workers Regulation, avant de disparaître dans les années 2000. Pour autant, la rémunération réglementée par la loi ne correspond pas à la définition que Shakti et les autres femmes habitant la côte ont du droit. L'État n'apparaît pas comme une institution de régulation du travail juste et légitime, parce qu'il ne garantit pas le droit à la subsistance tel qu'il est conçu par ces femmes. Cette notion du droit s'élabore d'après les conditions de reproduction actuelles de leur foyer, mais aussi d'après les mémoires des arrangements sociaux réciproques que les femmes de la côte avaient établis avec les propriétaires terriens avant le développement immobilier. Le fait que le service domestique se soit rapproché de la condition salariale n'a pas modifié la conception que les femmes habitant la côte ont de leur droit à assurer par leur travail la survie de leur foyer, que ce soit dans leur rapport aux employeurs de service domestique ou dans leur rapport à l'État.

Alors qu'à l'époque du travail dans la cour, «c'était une condition» de servir sans limite en termes de temps et de tâches en échange des ressources matérielles nécessaires à la subsistance, à présent que la paye de bonne est considérée comme insuffisante et injuste par rapport au revenu qu'elle doit apporter au foyer, de quelle manière les femmes sont-elles disposées à servir?

42. Entretien avec Shakti, Tamarin, 2 février 2019. 
Pour y répondre, il faut être attentif à la façon dont elles parlent de leurs employeurs et du service domestique que ceux-ci attendent. Prenons par exemple le discours d'Ananda, 60 ans, habitante de Tamarin:

Ananda: «Beaucoup de ceux chez qui j'ai travaillé sont domineurs. Là, j'ai un monsieur avec qui je travaille, domineur domineur. Très domineur. Vous voyez un Blanc, sa maison est blanche comme votre chaise. Il a deux chiens, il met ses chiens dans la maison. C'est domineur ça! Tous les jours, il dîne, il déjeune, dimanche, samedi, il fait bien la fête avec ses amis. Lundi, quand je vois la vaisselle là, j'ai envie de me sauver. Je vous dis franchement, ils sont bien domineurs. En plus ils ne cherchent pas à vous payer. Samedi, tu as besoin de travailler chez lui! Bien domineur.

Vous pensez que c'est partout pareil?

Partout pareil! Les étrangers, ils viennent d'ailleurs, ils font d'une autre façon. Quand ils voient comment les Mauriciens font, ils font pareil! Domineurs ${ }^{43}$.»

En qualifiant son employeur et, finalement, l'ensemble des employeurs de la côte, y compris les étrangers, de domineurs, Ananda livre sa représentation de la perpétuation du travail servile dévolu à la bonne dans le contexte actuel. Elle doit faire tout ce que l'employeur pourrait faire par lui-même (dont la cuisine et la vaisselle du week-end qui sont soulignées dans son discours) et même plus: c'est parce qu'elle est là pour nettoyer que les chiens peuvent rentrer dans la maison par exemple. Le travail de la bonne a pourtant objectivement changé depuis la disparition des cours et de leurs dépendances. Il aurait été incongru, dans les années 1960 et 1970, de qualifier ses patrons de domineurs parce qu'ils faisaient travailler le samedi: les bonnes n'avaient même pas le dimanche férié avant la promulgation de la loi. Le terme de domineur, employé par Ananda comme par d'autres femmes de l'enquête, désigne un employeur-type dont la pratique exigeant de la bonne un travail servile est jugée décalée par rapport à ce que le travail procure aujourd'hui comme ressources pour la subsistance du foyer de celle-ci. Le sens que les femmes employées comme bonnes attribuent à la condition du service domestique diverge désormais de celui que les employeurs attribuent au travail de bonne. Elles considèrent que les attentes de ces derniers sont illégitimes, parce que l'arrangement social pour le droit à la subsistance, qui maintenait le rapport de dépendance du travail dans la cour, a été rompu avec les transformations globales de l'économie de la côte.

43. Entretien avec Ananda, Tamarin, 25 février 2019. 
Politiser le travail domestique

\section{FAIRE SES CONDITIONS: ENJEUX ET EFFETS DE LA DISSIDENCE}

Puisque les femmes employées comme bonnes n'adhèrent pas vraiment à la condition du service domestique imposée par leurs employeurs aujourd'hui, certaines tentent de faire leurs conditions. L'expression créole désigne concrètement la pratique qui consiste à définir ses conditions de travail dans les relations interindividuelles avec leurs employeurs au moment de l'embauche. On a vu par exemple que Shakti parvenait à définir la rémunération de son travail avec son employeuse en fonction de l'évaluation de ses besoins pour sa subsistance. Elle s'appuie pour cela sur un barème de rémunérations pratiquées par les employeurs étrangers de la zone (français et sud-africains notamment) qui payent leur bonne près du double de la rémunération minimum réglementée par l'État (100 roupies de l'heure, soit deux euros cinquante). Parmi les femmes enquêtées, Shakti n'est pas la seule à faire ce calcul. Ce prix du service à l'heure tend à être généralisé et étendu aux employeurs qui ne font pas partie du cercle de ces étrangers, par des employées domestiques qui font leurs conditions à l'embauche. La définition de ses conditions porte aussi sur la nature des tâches et les horaires de travail. La plupart des femmes rencontrées refusent par exemple de faire la cuisine en plus du ménage quotidien comme il leur est souvent demandé. Une importante partie d'entre elles n'accepte pas non plus de travailler après 15 heures ou le samedi. Ces conditions établies dans le face-à-face avec les employeurs tendent à influer sur la forme que prennent les emplois domestiques de la côte, que ce soit au niveau de la rémunération, des horaires de travail ou de la spécialisation des tâches. Les quelques entretiens que j'ai pu mener avec des femmes travaillant comme bonnes dans d'autres zones de l'île montrent que ces conditions de travail obtenues par des femmes sur la côte entre Tamarin et Rivière Noire sont loin d'être caractéristiques du service domestique contemporain à l'île Maurice. Par quelles pratiques sociales ont-elles alors été créées et pour quels enjeux?

Certaines de mes interlocutrices parmi les plus jeunes sont entrées dans le service domestique après avoir travaillé à l'usine. Elles expliquent dans leur récit qu'elles ont quitté l'usine pour le travail de bonne parce que les horaires de leur travail précédent ne leur permettaient pas de consacrer le temps qu'elles auraient voulu à leur foyer, en particulier à leurs enfants. L'emploi du temps classique des machinistes consiste en effet à travailler de 9 heures à 18 heures du lundi au samedi, auquel s'ajoutent les heures supplémentaires obligatoires et le temps de transport pour rentrer chez soi ${ }^{44}$. Or tout un

44. Les zones industrielles, situées en ville, sont particulièrement éloignées du domicile des femmes de Tamarin et de Rivière Noire. 
ensemble d'activités liées à la prise en charge des enfants de la naissance à l'adolescence (garde, surveillance en dehors de l'école, veille sanitaire, loisirs, etc.) ne sont pas socialisées par des structures étatiques, ni complètement entrées dans la sphère marchande. Elles incombent aussi principalement aux femmes dans la division sexuelle du travail interne aux foyers de travailleurs. Des femmes rencontrées durant mon enquête disent donc avoir quitté les secteurs d'activité de l'overtime 45 (l'usine surtout, mais parfois aussi l'hôtellerie) et être entrées dans le service domestique, précisément dans l'espoir de négocier la valeur de leur force de travail dans la relation interindividuelle et directe avec les employeurs. Cette valeur équivaut tout autant au revenu essentiel à la subsistance de leur foyer qu'au temps de travail qu'elles estiment socialement nécessaire à la reproduction de leur propre foyer. Le niveau de rémunération et la limite du temps employé au service domestique sont donc les éléments cruciaux de la négociation de ses conditions face aux employeurs.

L'aboutissement de cette négociation ne repose néanmoins pas que sur l'habileté individuelle de femmes à faire leurs conditions dans le face-à-face avec leurs employeurs. D'ailleurs c'est par l'emploi du pronom «nous» que des femmes habitantes de la côte me racontent leurs pratiques au travail: «Nou fer nou bann kondisyon.» Qui est ce «nous » et comment s'organise-t-il? Les interlocutrices qui assument cette pratique sociale dans leur discours appartiennent à des générations différentes. Elles sont mariées ou séparées, avec des enfants d'âges divers. Créoles ou hindoues, certaines ont toujours travaillé dans la cour, d'autres ont coupé la canne dans la plantation, d'autres encore ont été ouvrières du textile avant d'entrer dans le service domestique. Elles sont nées sur la côte ou y vivent depuis l'âge adulte (souvent depuis leur mariage). Autrement dit, aucune catégorie de classement statistique ne permet de circonscrire le groupe social en train de se former ${ }^{46}$ autour des femmes de la côte qui font leurs conditions sur le marché des emplois domestiques. Il faut au contraire examiner ce qu'exprime ce «nous » pour les femmes qui s'intègrent dans cette identité collective. Prenons par exemple le discours de Yolda, 40 ans, habitante de Rivière Noire et employée dans deux maisons:

Yolda: «Vous savez, il y a des femmes, elles ont fait 20 ans, 25 ans avec les mêmes personnes. Elles ne veulent pas changer. Mais après ça, vous savez, il y a des femmes qui viennent de loin, quand elles viennent, elles finissent par gâter notre prix du travail, c'est-à-dire le prix qu'on a réussi à avoir avec les patrons. On n'est pas des femmes paresseuses nous, c'est

45. Avec heures supplémentaires.

46. Voir J. Rancière, «Citoyenneté, culture et politique», in M. Elbaz et D. Helly (dir.), Mondialisation, citoyenneté et multiculturalisme, Paris/Québec, L'Harmattan/Presse de l'Université Laval, 2000, p. 55-68. 
Politiser le travail domestique

pas qu'on ne veut pas travailler et qu'on va s'asseoir dans la maison en attendant d'avoir quelque chose, non. Mais nous on ne travaille pas pour n'importe quelles conditions ${ }^{47}$.»

Le «nous» formulé par Yolda s'oppose d'abord aux femmes qui acceptent les conditions de travail imposées par leurs employeurs. Ces dernières sont d'une part celles qui travaillent depuis de longues années pour une même famille et qui sont encore enfermées dans un rapport de service. Celles-ci bénéficient peut-être encore d'arrangements sociaux particuliers avec leurs employeurs, mais ils ne sont plus caractéristiques des rapports plus globaux que les employées domestiques entretiennent avec les employeurs de services résidents des villas. Les femmes qui acceptent les conditions des employeurs s'incarneraient d'autre part dans les bonnes venues des villes pour travailler sur la côte. Le "nous» des femmes qui font leurs conditions tend donc, ici et maintenant sur la côte sud-ouest, à se fédérer autour des femmes qui habitent sur la côte. Ce sentiment de faire groupe de la part de femmes définissant leur «prix du travail » n'est pas déterminé par leur origine géographique, puisque certaines, comme Shakti ou Yolda, ne sont même pas nées sur la côte. Il est en revanche matériellement soutenu par les espaces habités qui subsistent de la vie des travailleurs de la côte, où les femmes se rencontrent et discutent de leurs manières de négocier les tâches, les horaires de travail et les taux de rémunération. Solange, Yolda, Shakti et Ananda, bien que ne vivant pas dans la même cité, se connaissent et échangent aux coins de rues, dans des petites boutiques, dans des lieux de prières ou aux moments de communion (comme les mariages) qui appartiennent aux espaces-temps réduits et ségrégués, mais autonomes et appropriés, de la vie sociale des travailleurs de la côte. En l'absence d'une organisation concrète autour d'un syndicat ou d'une association, ces femmes finissent néanmoins par former un groupe politique des employées domestiques qui font leurs conditions à partir d'une appartenance commune et d'espaces de sociabilité hérités des institutions sociales produites par les travailleurs de la côte.

Il y a enfin un autre aspect de l'investissement des femmes dans l'entretien de la vie sociale des travailleurs de la côte qui les arme de capacités de négociation de leurs conditions de travail dans le service domestique: le travay pou mo mem. Le travail pour moi-même désigne avec leurs mots une activité artisanale réalisée chez soi, comme la confection de plats ou de gâteaux, dont les produits sont vendus de manière formelle ou informelle sur le bord de la route et souvent devant leur maison. La plupart des consommateurs de leurs produits sont donc les habitants des cités ou des autres enclaves

47. Entretien avec Yolda, Rivière Noire, 7 mars 2019. 
résidentielles des travailleurs de la côte. Les employées domestiques qui se lancent dans ces petits commerces obtiennent un revenu supplémentaire qui leur permet de négocier plus facilement leurs conditions face aux employeurs, car elles réduisent par cette activité leur dépendance financière aux emplois domestiques. Le revenu de trois heures par jour d'un travay pou mo mem en cuisine, par exemple, équivaut environ à la rémunération de quatre heures de service domestique par jour pour certaines de mes interlocutrices. L'ajout du travay pou mo mem à leur journée de service les conduit aussi à réduire le temps alloué à la vente de leur force de travail pour le service domestique aux résidents des villas de la côte. On touche là à l'enjeu principal qui consiste à faire ses conditions sur le marché des emplois domestiques. Le groupe des femmes habitant la côte ne vise pas tant à améliorer ses conditions de travail pour les faire se rapprocher de conditions salariales, contractuelles et se démarquant du rapport de dépendance d'autrefois. Il agit dans le but de conserver, tant bien que mal, un droit à la subsistance et à la reproduction de leur foyer sur la côte, dans une autonomie relative vis-à-vis du travail de bonne pour des employeurs, pour des domineurs, pour les avatars des Blancs qui étaient auparavant servis par les travailleurs de la côte.

Le type de petite entreprise que représente l'activité artisanale de femmes dans les cités et dans les autres espaces d'habitat modeste est certes encouragé par l'État et par les promoteurs du développement immobilier, afin de maintenir la solvabilité des foyers de travailleurs confrontés au chômage masculin ${ }^{48}$. Cette politique économique entend également externaliser les risques liés à la restructuration de l'économie sucrière ainsi que les coûts de reproduction de la force de travail sur les femmes ${ }^{49}$. En effet, ces dernières produisent de la sorte une nourriture bon marché pour l'ensemble des travailleurs dont les salaires ne permettent que de manière limitée d'acheter dans les supermarchés situés à proximité. Mais si l'on tient compte du sens que les femmes produisent sur cette activité artisanale, le travay pou mo mem tel qu'elles la désignent, apparaît, dans sa mise en rapport discursive avec le service domestique, comme ce qui s'oppose au statut d'employée pour un propriétaire immobilier usager de services domestiques. Or ce statut d'employée s'inscrit, dans leur manière de penser, dans une continuité avec

\footnotetext{
48. Sur la microfinance pour restaurer la solvabilité des pauvres, voir par exemple L. Bazin, «Le travail: un phénomène politique complexe et ses mutations conjoncturelles», Politique africaine, $\mathrm{n}^{\circ} 133,2014$, p. 7-23.

49. P. Neveling, «Beyond Sites and Methods: The Field, History, Global Capitalism», in S. Coleman, S. B. Hyatt et A. Kingsolver (dir.), Routledge Companion to Contemporary Anthropology, Londres, Routledge, 2016, p. 72-91; W. A. Dunaway, «The Double Register of History: Situating the Forgotten Woman and her Household in Capitalist Commodity Chains ", Journal of World-Systems Research, vol. $7, \mathrm{n}^{\circ} 1,2001$, p. 2-29.
} 
Politiser le travail domestique

le service auprès des Blancs propriétaires des cours et des tablismans d'autrefois. Le travay pou mo mem devient, par les significations politiques données au travail, un outil de transformation des conditions du service domestique. De plus, il recrée un lien entre l'organisation de la reproduction des foyers de travailleurs et le territoire de la côte ${ }^{50}$. Ces petites commerçantes réaffirment de fait des modes d'alimentation internes et exclusifs aux travailleurs de la côte, bien qu'ils soient retranchés aux quelques enclaves de leurs habitats. Enfin, il est également une source de revenus grâce auxquels les femmes réélaborent les rapports de genre internes du foyer, parce qu'elles utilisent la caractéristique incertaine et fluctuante des revenus rapportés par cette activité pour en dissimuler une partie à la connaissance de leur mari et en faire un usage personnel. Les femmes ne cherchent donc pas que la survie de leur foyer dans leur rapport politique au travail, mais également une mainmise sur la production sociale de leur existence, à la fois dans et en marge du développement immobilier de la côte.

Lorsque les femmes habitant la côte n'ont pas les conditions qu'elles souhaitent définir dans le service domestique, elles décident de quitter certains de leurs employeurs, ou bien elles ne seront pas les bonnes accueillantes, efficaces, fidèles et serviles attendues. Leurs capacités de négociation, acquises grâce aux regroupements sociaux, à la signification divergente donnée au travail et à l'enracinement social dans la terre côtière, entravent de fait l'expansion du développement immobilier. Les employeurs de service domestique et les promoteurs immobiliers cherchent par conséquent à freiner les effets de ces pratiques dissidentes. Pour ce faire, ils affublent les femmes de la côte qui refusent d'accepter les conditions du service domestique d'une image qui les dégrade et d'un comportement immoral: la paresse. La crédibilité de la dégradation morale de leur groupe est assurée par l'association de la paresse à une catégorie ethnique issue de la production idéologique coloniale, celle de Créole, affiliée aux descendants d'esclaves ${ }^{51}$. Ainsi, bien qu'Hindoues d'après leurs pratiques religieuses, Shakti et Ananda sont, du fait de leurs dispositions dissidentes par rapport aux conditions du service domestique de leurs employeurs, globalement assimilées aux Créoles «paresseuses » et «arriérées » de la côte. Ces représentations assorties d'un jugement moral légitiment donc le recours de la part d'employeurs à la force de travail de

50. I. Wallerstein, «Les structures du foyer domestique et la constitution de la force de travail dans l'économie-monde capitaliste», in É. Balibar et I. Wallerstein (dir.), Race, nation, classe. Les identités ambiguës, Paris, La Découverte, 2018, p. 175-183.

51. Sur la relation des formations ethniques à un code moral, voir B. F. Williams, «A Class Act: Anthropology and the Race to Nation across Ethnic Terrain», Annual Review of Anthropology, vol. 18, 1989 , p. 401-444. 
femmes des villes des plateaux centraux. Celles-ci n'ont en effet pas forcément les mêmes ressources sociales pour résister au «service à la Mauricienne» qui leur est enjoint. La catégorisation ethnique par le biais de laquelle le marché des emplois domestiques de la côte est réorganisé est alors le résultat, plutôt que la cause, des tensions produites par les femmes de la côte dans leurs rapports aux employeurs de service domestique, et plus globalement dans leurs rapports aux propriétaires terriens, aux promoteurs immobiliers et à l'État.

L'examen de l'économie morale des femmes employées domestiques habitant la côte sud-ouest mauricienne propose une analyse du service domestique contemporain qui se distingue sur bien des points d'une approche qui décrit ce travail comme un élément profondément conservateur de la hiérarchie sociale entre les classes, les races $^{52}$ et les genres ${ }^{53}$. L'analyse des discours de mes interlocutrices a permis de voir que, derrière les rapports de dépendance et l'attitude servile exigée du temps du travail dans la cour, s'élaboraient des arrangements sociaux réciproques, une norme du droit et une organisation sociale de la subsistance des travailleurs dans une relative autonomie vis-à-vis des propriétaires terriens. Les mémoires de cette économie morale du rapport de dépendance sont constitutives d'une interprétation, par des femmes employées domestiques, de la détérioration des conditions matérielles d'existence de leur foyer et de la perte de leurs droits avec les transformations de l'économie de plantation, l'expansion de l'économie de marché et l'invasion du développement immobilier. Bien loin de considérer le travail «salarié» de service domestique et le développement de la côte comme des facteurs d'amélioration de leurs conditions de travail et de vie, elles construisent alors leurs pratiques sociales en dissidence par rapport à l'ensemble des bénéficiaires de services domestiques. Elles perpétuent de la sorte les luttes anciennes de femmes pour la survie de leur foyer et pour la production sociale de leur existence à la marge ${ }^{54} \mathrm{du}$ contrôle des propriétaires des moyens de production et de la terre. Il s'agit à la fois des employeurs ayant acquis des villas, des anciens propriétaires terriens, des nouveaux promoteurs immobiliers et de l'État.

Le spectre politique dans lequel ces femmes inscrivent leurs rapports de travail aux employeurs de service domestique ne peut donc être appréhendé sans adopter une méthodologie qui explore leur subjectivité en considérant

52. Races en tant que construits sociaux de l'idéologie raciste.

53. Voir par exemple J. Rollins, «Entre femmes...», art. cité.

54. S. W. Mintz et R. Price, The Birth of African-American Culture..., op. cit. 
Politiser le travail domestique

l'ensemble des espaces sociaux (et leur profondeur mémorielle) dans lesquels elles sont impliquées ${ }^{55}$ pour la survie de leur foyer. Ces espaces sont séparés, dans leurs discours et dans leurs pratiques, en deux: celui du travail pour les dominants possédant la terre et le capital, et celui du travay pou mo mem dans le cadre des rapports internes aux travailleurs de la côte.

Leur travail ne sert alors que partiellement les intérêts du développement immobilier et la reproduction, tant de la hiérarchie sociale, que de la force de travail censée constituer le «service à la Mauricienne». Ce n'est qu'en tenant compte de leurs manières de penser le travail, leurs droits, les transformations économiques et les rapports sociaux passés que l'on peut enfin comprendre la façon dont les rapports d'exploitation et de domination sont réagencés ${ }^{56}$. Dans la dynamique des rapports sociaux, la dissidence des femmes employées domestiques de la côte se situe en effet entre le travail dépendant et son évolution vers des emplois domestiques, entre l'économie de plantation et sa transformation en un développement immobilier de luxe, entre l'idéologie raciste d'autrefois et la (re)production permanente des catégorisations ethniques aujourd'hui

Colette Le Petitcorps Institute of Social Sciences, University of Lisbon

\section{Abstract \\ Dissidence in Domestic Service. The Moral Economy of Women, Housemaids in Mauritius}

The female labour force in domestic service has become a main factor of production for property development pursued under the restructuration of the Mauritian sugar economy, especially on the coast. This paper examines the subjective meanings and social practices by which domestic workers living on the coast deal with the transformations of work and land according to the issues of their own household's reproduction. The analysis of their moral economy gives a special insight on the political subject they create from domestic service but also beyond the scope of wage labour. 